\title{
Modelling and Control of Helicopter Robotic Landing Gear for Uneven Ground Conditions
}

\author{
Daniel Melia Boix ${ }^{1}$, Keng Goh ${ }^{2}$ and James McWhinnie ${ }^{3}$ \\ School of Engineering and Built Environment, Edinburgh Napier University, Edinburgh, EH105DT, United Kingdom \\ 11.meliaboix@napier.ac.uk, ²k.goh@napier.ac.uk and 3j.mcwhinnie@ napier.ac.uk
}

\begin{abstract}
This paper proposes a method to investigate into helicopter landing on uneven terrain by means of using a scaled articulated robotic landing gear. A mathematical model of an articulated robotic landing gear that adapts to uneven ground conditions is considered. The model consists of a planar landing gear composed of two legs connected by a base and a skid at each end. Each skid has two degrees of freedom with PID joint controllers to provide stability while landing. A combination of Lagrange and Newton-Euler techniques is used to model the system dynamics. This work also includes a model of the ground interaction, a thrust controller and a level controller to maintain stability while landing. Experimental results with a laboratorybuild scaled prototype are included and compared with the simulations.
\end{abstract}

Keywords-PID control, robotic landing gear, nonlinear dynamic model, contact model, level controller, robot prototype

\section{INTRODUCTION}

Helicopters are widely used in search and rescue missions, disaster areas or mountainous environments because of their ability to access remote or unprepared areas that are not accessible by any other means. This is due mainly to their ability to perform hovering and vertical landing/takeoff manoeuvres. However, they usually incorporate fixed landing gear systems like skids or wheels, which limit their ability to land on irregular terrain.

A number of early publications suggested several solutions to cope with the problem of landing on sloped surfaces using hydraulic or mechanical systems to adapt the position of wheels/legs to the ground conditions [1], [2], but with limited study available since then. A recent example of robotic landing gear was developed by the Georgia Institute of Technology under the DARPA's Mission Adaptive Rotor (MAR) program [3] consisting of four articulated robotic legs that adapt their position to ensure that the helicopter stays level during landing. Despite practical tests have been carried out [4], limited information has been made available about its system dynamics and control strategy.

Dynamics modelling techniques are generally divided into two main categories, the ones that are based in NewtonEuler equations, and the ones based on analytical mechanics approaches, like Lagrange method [5]. When modelling multibody systems, a common practice is to use an abstract, simplified model of the robot to reduce the model's complexity. A mixed approach is found in [6] and [7] where the authors use a modelling technique for quadruped robot locomotion based on decoupling the body and legs. For body position and attitude, a point-mass model of the Centre of
Mass (CoM) is analysed and the external forces are computed. Then the joint torques are calculated using a single leg dynamics model. The whole body model is constructed by coupling the equations of the floating-base body and each of the legs attached to it.

Examples are also found in the field of Mobile Manipulating Unmanned Aerial Vehicles (MM-UAV), a recent research line that consists on attaching one or several robot-arms to the body of a rotorcraft in order to interact with the environment and perform manipulation tasks [8], [9], [10].

In this paper, a planar dynamic model of a two-leg landing gear and the control strategy to keep the helicopter base stable when landing on irregular terrain is presented. In Section II, a kinematic and dynamic model of a single leg are derived using the Lagrange method and then, the position and inclination of the base are computed applying the NewtonEuler equations to obtain the Centre Of Mass dynamics. The whole system model is built by coupling the base model with two single-leg models.

In Section III a landing scenario is designed including a simplified model of a thrust force to control the landing velocity and a contact model to emulate the ground-leg reaction and friction forces. In Section IV a level controller is also designed to adapt the position of the legs to the ground conditions. Finally, in Sections V and VI a laboratory-built prototype of the landing gear is presented and the experimental results are compared with the software simulations.

\section{System KinEMATICS AND DYNAMICS}

\section{A. Single-leg dynamic model}

In this section the mathematical model of a robot leg is presented. Each leg has 2 links and two revolute joints at the hip and knee with its axes of rotation perpendicular to the $\mathrm{XY}$ plane, therefore the system is two-dimensional or it has 2DOF. The hip and knee angles are represented by $\theta_{1}$ and $\theta_{2}$ respectively with its origin as shown in Figure 1 and positive in the anti-clockwise direction. The length of the two links is represented by $L_{1}$ and $L_{2}$ respectively and their masses are $m_{l}$ and $m_{2}$. For simplification, the masses are considered to be concentrated at the end of each link.

The equations of motion of the single leg are derived using the Lagrange-Euler formulation as it presents a more systematic derivation and provides a closed-form expression [11]. This approach analyses the system in terms of work and 


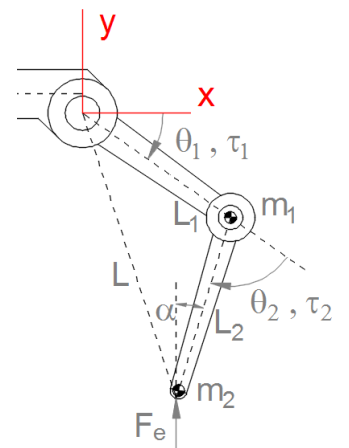

Figure 1. Sketch of a robotic leg with relevant parameters. It shows dimensions and masses of links, joint angles and torques and external forces.

energy and it's based on the Lagrangian function $(\mathcal{L})$ as the difference between the kinetic energy $\left(K_{E}\right)$ and the potential energy $\left(P_{E}\right)$ of the system as

$$
\mathcal{L}=K_{E}-P_{E}
$$

The equations of motion are obtained by solving the Euler-Lagrange equation:

$$
\frac{d}{d t}\left(\frac{\partial \mathcal{L}}{\partial \dot{\theta}_{n}}\right)-\frac{\partial \mathcal{L}}{\partial \theta_{n}}=\tau_{i}
$$

where $\theta_{n}$ is the joint angle of the link $n$, and $\tau_{i}$ represents the torque applied to the joint $i$.

The net torque acting on the hip and knee joints is given by the following expressions [12]:

$$
\begin{aligned}
\tau_{T 1}=\left(\left(m_{1}+m_{2}\right)\right. & L_{1}^{2}+m_{2} L_{2}^{2} \\
+ & \left.2 m_{2} L_{1} L_{2} \cos \theta_{2}\right) \ddot{\theta}_{1} \\
+ & \left(m_{2} L_{2}^{2}+m_{2} L_{1} L_{2} \cos \theta_{2}\right) \ddot{\theta}_{2} \\
& -m_{2} L_{1} L_{2}\left(2 \dot{\theta}_{1} \dot{\theta}_{2}+\dot{\theta}_{2}^{2}\right) \sin \theta_{2} \\
& +\left(m_{1}-m_{2}\right) g L_{1} \cos \theta_{1} \\
& +m_{2} g L_{2} \cos \left(\theta_{1}+\theta_{2}\right) \\
\tau_{T 2}=m_{2} L_{2}^{2} \ddot{\theta}_{2}+ & \left(m_{2} L_{2}^{2}+m_{2} L_{1} L_{2} \cos \theta_{2}\right) \ddot{\theta}_{1} \\
& +m_{2} L_{1} L_{2} \dot{\theta}_{1}^{2} \sin \theta_{2} \\
& +m_{2} g L_{2} \cos \left(\theta_{1}+\theta_{2}\right)
\end{aligned}
$$

The total joint torque, $\tau_{T n}$, is the sum of the torque produced by the joint actuator, $\tau_{n}$, and the torque induced by the external forces acting on the leg, $F_{e}$. In this model, the only external forces that are considered acting on the legs are the ground reaction and friction forces. The torque induced in the joints by the external forces is computed using the transpose of the Jacobian matrix

$$
\begin{aligned}
\tau_{T} & =\tau+J^{T} \cdot F_{\text {ext }} \\
J^{T} \cdot F_{\text {ext }} & =\left[\begin{array}{ll}
\frac{\partial x_{2}}{\partial \theta_{1}} & \frac{\partial y_{2}}{\partial \theta_{1}} \\
\frac{\partial x_{2}}{\partial \theta_{2}} & \frac{\partial y_{2}}{\partial \theta_{2}}
\end{array}\right] \cdot\left[\begin{array}{l}
F_{x} \\
F_{y}
\end{array}\right]
\end{aligned}
$$

The position of the foot is calculated using Forward Kinematics equations, which provide the X-Y foot coordinates (with respect to the hip joint) as a function of the joint angles

$$
\begin{gathered}
x_{2}=L_{1} \cos \theta_{1}+L_{2} \cos \left(\theta_{1}+\theta_{2}\right) \\
y_{2}=-L_{1} \sin \theta_{1}-L_{2} \sin \left(\theta_{1}+\theta_{2}\right)
\end{gathered}
$$

The Inverse Kinematics equations provide the joint angles as a function of the $\mathrm{X}-\mathrm{Y}$ foot coordinates

$$
\begin{gathered}
\theta_{1}=\tan ^{-1}\left(\frac{x_{2}}{y_{2}}\right)+\cos ^{-1}\left(\frac{L_{1}^{2}+L^{2}-L_{2}^{2}}{2 L_{1} L}\right)-90^{\circ} \\
\theta_{2}=\cos ^{-1}\left(\frac{L_{1}^{2}+L_{2}^{2}-L^{2}}{2 L_{1} L_{2}}\right)-180^{\circ}
\end{gathered}
$$

Figure 2 shows the control diagram of a single leg. The position of the leg is controlled by introducing the desired $\mathrm{X}$ $\mathrm{Y}$ foot coordinates $\left(x_{2}, y_{2}\right)$. The Inverse Kinematics block calculates the reference joint angles which are introduced as a setpoint into PID joint controllers that generate appropriate joint actuator torques and introduce them into the Dynamic Model of the leg. The Dynamic model reproduces the motion of the leg and calculates the current joint angles which are used as a feedback to the joint controllers. Using Forward Kinematics, the current $\mathrm{X}-\mathrm{Y}$ foot position is obtained.

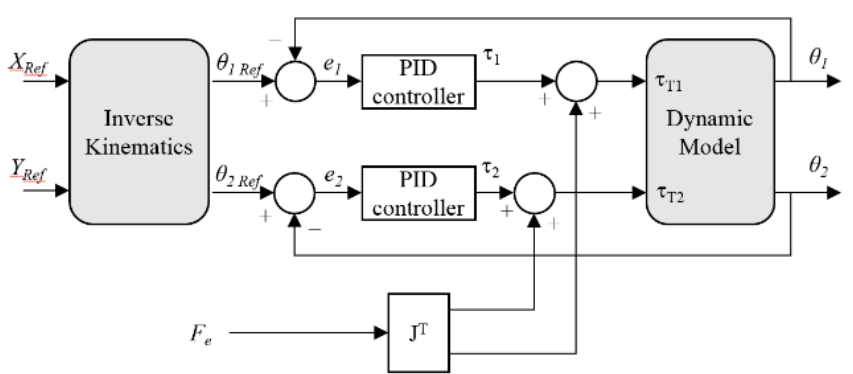

Figure 2. Control block diagram of a single leg

\section{B. Centre of Mass dynamics}

The complete landing gear model, is a multibody system formed by two robotic legs connected through a base link. The position and inclination of the base with respect to the ground is computed by using a point-mass model of the landing gear. All the different bodies that form the system are reduced to a single point that has a rotational inertia, $I$, and a total mass, $m$, which emulate those of the whole system and is located at the Centre of Mass of the landing gear. For simplification purposes, the CoM is considered to be at the centre of the base link, as the mass of the legs is negligible compared to the mass of the base.

The landing scenario includes a thrust force model to control the descending speed and a ground contact model.

Newton-Euler equations are used to calculate the CoM linear and angular acceleration ( $\ddot{x}, \ddot{y}$ and $\ddot{\theta}$ respectively) and to obtain the $\mathrm{x}$ and $\mathrm{y}$-position, and roll angle

$$
\begin{gathered}
\sum F_{y}=m \ddot{y}=m g-F_{\text {Thrust }}-F_{y_{L}}-F_{y_{R}} \\
\sum F_{x}=m \ddot{x}=F_{x_{L}}+F_{x_{R}} \\
\sum M_{C o M}=I \ddot{\theta}=F_{y_{L}} x_{L}-F_{y_{R}} x_{R}+F_{x_{L}} x_{L}+F_{x_{R}} x_{R}
\end{gathered}
$$


where $m$ is the system's total mass, $g$ is the gravity acceleration, $I$ is the system's inertia and $F_{\text {Thrust }}$ is the thrust controller force. $F_{y_{L}}$ and $F_{x_{L}}$ are the sum of the forces acting on the left foot in the $\mathrm{y}$ and $\mathrm{x}$ direction, and $x_{L}$ and $y_{L}$ are the distances from the CoM to the left foot in the $\mathrm{x}$ and $\mathrm{y}$ directions. $F_{y_{R}}, F_{x_{R}}, x_{R}$ and $y_{R}$ are the equivalent on the right foot. The roll angle is considered positive when rotates in the clockwise direction. The thrust controller is a simplified version of the one used in [4] where the only aim is to control the descent rate

$$
F_{t h}=C \cdot\left(\dot{y}_{r e f}-\dot{y}\right)+m g
$$

where $\mathrm{C}$ is a constant.

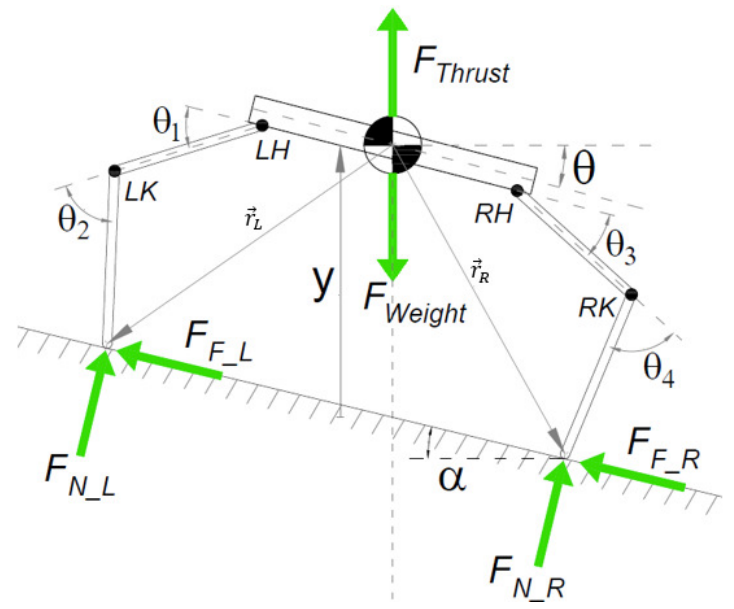

Figure 3. Sketch of whole landing gear. In the left side, the upper and lower links are Link 1 and 2 , and hip $(L H)$ and knee $(L K)$ angles are $\theta_{1}$ and $\theta_{2}$ respectively. In the right side, the upper and lower links are Link 3 and 4 , and hip $(R H)$ and knee $(R K)$ angles are $\theta_{3}$ and $\theta_{4}$ respectively.

\section{GROUND CONTACT MODEL}

\section{A. Normal Force}

To simulate the ground contact forces during landing, a compliant contact model composed by a spring-damper system has been used. The main advantages of this approach are its good performance and straightforward implementation, and the fact that there are lots of examples in literature where it was successfully implemented as reported in [13], [14], [15] and [16].

Equation (15) represents the normal force to the ground when a leg tip lands, where $y$ is the current leg tip vertical coordinate (normal to the ground), $k$ is the spring elastic constant, and $b$ is the damping ratio. The ground reaction force resists the leg tip penetrating the ground surface and it is proportional to the amount of ground penetration (spring component) and the rate of penetration (damper component).

$$
F_{N} \begin{cases}-y \cdot k-\dot{y} \cdot b & \text { if } y \leq 0 \\ 0 & \text { if } y>0\end{cases}
$$

The normal force is zero before the impact and starts to make effect at the moment of touchdown, when $y=0$. At this point the force will peak due to the effect of the damper force because the foot is penetrating at landing velocity. After a few bounces the velocity goes to 0 and the foot position settles down to a certain ground penetration due to the effect of the spring force. Positive velocities would produce a damper force that tends to hold or stick the foot to the ground. This has to be avoided as it is physically incorrect and the system has to be allowed to bounce.

\section{B. Friction force}

Apart from the ground reaction force normal to the ground, friction force is also modelled to simulate the sliding of the system when landing on slopped surface. In [16] and [17], the authors present different friction force models. The model used in this paper is a variation of the Coulomb friction model for its simplicity and approximation to reality.

Friction force has opposite direction to the sliding velocity, and is a non-linear function of the relative velocity and position of the two contacting surfaces. At the moment of touchdown, the friction force is in the "sticking" region, and it is modelled as a spring-damper system and it's proportional to the tangential velocity and displacement of the foot. If the velocity and displacement keep growing until the friction force exceeds the maximum static friction force, $F_{F_{-} S}$, then the contact model switches to "slip" mode and the friction force is equal to the dynamic friction force, $F_{F_{-} D}$

$$
\begin{gathered}
F_{F} \begin{cases}-\left(x-x_{0}\right) \cdot k-\dot{x} \cdot b & \text { if } F_{F}<F_{F_{-} S} \\
F_{F_{-} D} & \text { if } F_{F} \geq F_{F_{-} S}\end{cases} \\
F_{F_{-} S}=\mu_{S} F_{N} \\
F_{F_{-} D}=\mu_{D} F_{N}
\end{gathered}
$$

where $\mu_{\mathrm{S}}$ and $\mu_{\mathrm{D}}$ are the static and dynamic friction coefficients, and $x_{0}$ is the $\mathrm{x}$ foot coordinate at the moment of ground contact. The distance $x-x_{0}$, is measured in the tangential direction to the ground.

\subsection{Normal and tangential forces on slopped ground}

The positions and velocities of the CoM and the feet are referred to the world coordinate frame XY axis (in the horizontal and vertical direction), but when dealing with slopped surfaces, in order to calculate ground reaction and friction forces, the positions and velocities of the feet have to be referred to the ground normal and tangential axis

$$
\begin{gathered}
x_{t}=x_{h} \cos \alpha+y_{v} \sin \alpha \\
y_{n}=-x_{h} \sin \alpha+y_{v} \cos \alpha
\end{gathered}
$$

where $x_{h}$ and $y_{v}$ are the horizontal and vertical coordinates and $x_{t}$ and $y_{n}$ are the tangential and normal coordinates.

After the normal force, $F_{N}$, and friction force, $F_{F}$, are calculated, its horizontal and vertical components are calculated as:

$$
\begin{gathered}
\vec{F}_{N}=F_{N} \vec{n}=-F_{N} \sin \alpha \vec{x}+F_{N} \cos \alpha \vec{y} \\
\vec{F}_{F}=F_{F} \vec{t}=F_{F} \cos \alpha \vec{x}+F_{F} \sin \alpha \vec{y}
\end{gathered}
$$

To compute the effect of the ground reaction and friction forces on the leg model, the sum of the vertical and horizontal forces needs to be calculated as: 


$$
\begin{gathered}
F_{x}=-F_{N} \sin \alpha+F_{F} \cos \alpha \\
F_{y}=F_{N} \cos \alpha+F_{F} \sin \alpha
\end{gathered}
$$

\section{LEVEL CONTROLLER}

The level controller $(L C)$ takes the inclination of the base as an input and generates a signal to increase/decrease the height of both legs in order to make the inclination angle equal to 0 and maintain the base horizontal.

The algorithm considered to control the height of the legs is a PI with the following control law equation:

$$
y_{\text {inc }}=K_{P} e(t)+K_{I} \int_{0}^{t} e(t)
$$

where $y_{i n c}$ is the height increase/decrease of both legs, $e(t)$ is the error between the measured angle and the horizontal position, and $K_{P}$, and $K_{I}$ are proportional and integral gains respectively.

The leg height is adjusted by adding/subtracting the height increment to the initial height of each leg

$$
\begin{aligned}
y_{\text {Left Goal }} & =y_{\text {Left }}-y_{\text {inc }} \\
y_{\text {Right Goal }} & =y_{\text {Right }}+y_{\text {inc }}
\end{aligned}
$$

So, if the angle is positive, the controller will extend the right leg and retract the left leg, and if the angle is negative, will do the opposite.

\section{PRototyPe DesigN}

Figure 4 shows the laboratory-fabricated prototype in order to carry out the experimental tests. The robotic legs include two Dynamixel AX-18 smart servo motors each, at the hip and knee joints. These motors provide position and load feedback to the system. For motors position control the default manufacturing settings have been used. The prototype is equipped with an on-board microcontroller ArbotiX-M and an Inertial Measurement Unit (IMU) sensor MPU-6050 with 3 -axis accelerometer and gyroscope. The microcontroller includes TTL ports for direct connection with the servos and I2C bus to interface with the IMU unit. The power is supplied by an external source of 12 VDC.

The code introduced in the microcontroller gets the base inclination from the IMU unit using a Kalman filter and checks the load feedback from both hip servos. If a load threshold is reached the system detects that a leg has made ground contact and the level controller is turned on. The LC uses servos position feedback and kinematics equations to get/set the XY coordinates of the feet. To simulate a helicopter landing, an external weight is added to the system during the performing of the tests and the system is lowered down using a rope and a pulley. Should the robotic legs behave abnormally, such as not fully extend/retract to the desired setpoint, the position feedback signal from the motors can be calibrated to indicate the fault and subsequently, the system will be switched off under the current experimental setup.

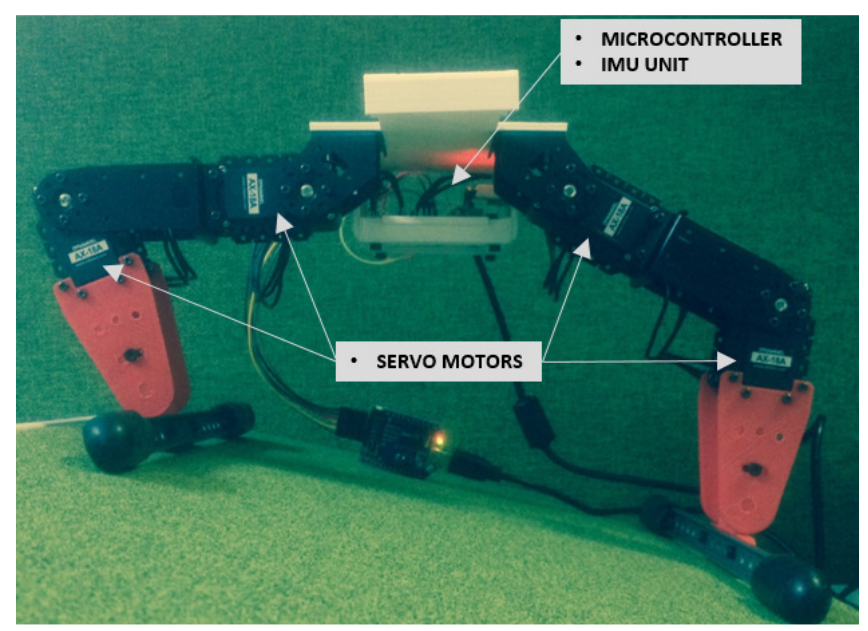

Figure 4. Image of the laboratory-built landing gear prototype

The physical parameters of the prototype are described in the table below.

Table 1. Prototype physical parameters
\begin{tabular}{|l|l|l|}
\hline Parameter & Symbol & Value \\
\hline Upper/Lower leg mass & $m_{1} / m_{2}$ & $0.1 / 0.15 \mathrm{~kg}$ \\
\hline Upper/Lower leg length & $L_{1} / L_{2}$ & $0.0935 / 0.1045 \mathrm{~m}$ \\
\hline Base length & $D$ & $0.1 \mathrm{~m}$ \\
\hline $\begin{array}{l}\text { Total system mass } \\
\text { (external weight included) }\end{array}$ & $m$ & $3 \mathrm{~kg}$ \\
\hline Motor max torque & $T_{\max }$ & $18 \mathrm{kfg} \cdot \mathrm{cm} / 1.76 \mathrm{Nm}$ \\
\hline
\end{tabular}

\section{SimULATION AND EXPERIMENTAL RESUltS}

\section{A. Simulation parameters}

A MATLAB/Simulink model is designed to simulate a landing test. The weights, dimensions and motors maximum torque used in the simulations are the same as the prototype. The system total inertia used is $0.08 \mathrm{~kg} / \mathrm{m}^{2}$, and the spring and damper coefficients for the ground contact model are 1500 $\mathrm{kg} / \mathrm{s}^{2}$ and $30 \mathrm{~kg} / \mathrm{s}$ respectively. The landing scenario is set at a rate of descent of the landing gear of $0.5 \mathrm{~m} / \mathrm{s}$ and the slope of the ground of $12^{\circ}$.

There are several tuning methods available for PID controller [18]. However, due to the complexity of the model, the controllers are tuned manually by trial and error to obtain the desired performance. The controller parameters are summarised in Table 2.

\section{Table 2. Controllers' parameters}

\begin{tabular}{|c|c|c|}
\hline Controller Gain & Joint controllers & $\begin{array}{c}\text { Level } \\
\text { Controller }\end{array}$ \\
\hline $\boldsymbol{K}_{\boldsymbol{P}}$ & 75 & 0.0025 \\
\hline $\boldsymbol{K}_{\boldsymbol{I}}$ & 40 & 0.025 \\
\hline $\boldsymbol{K}_{\boldsymbol{D}}$ & 4.5 & -- \\
\hline
\end{tabular}

\section{B. Simulation results}

The simulation and practical results are shown in Figures 5-7 where the software simulation and the landing test with the prototype are compared. 


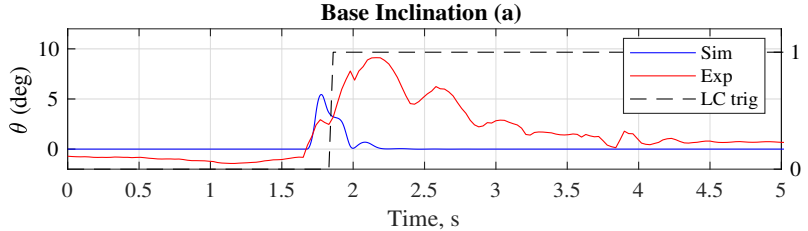

Left leg height $(b)$

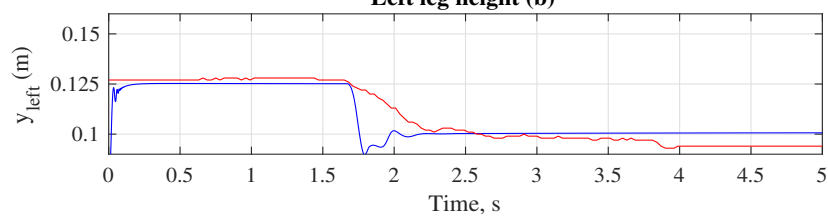

Right leg height (c)

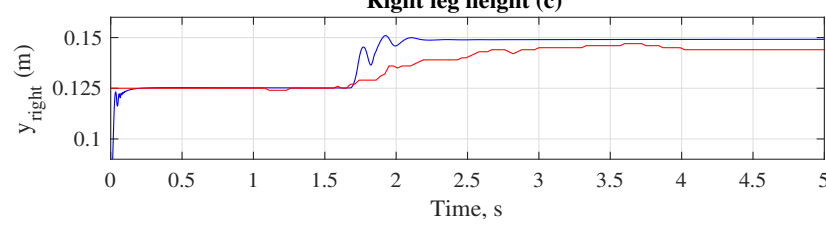

Figure 5. Base inclination (a), and vertical length of left leg $(b)$ and right leg (c). Blue plots represent the simulations and red ones represent the experimental test. Dotted line represents the level controller trigger.

Figure 5 shows that touchdown occurs at 1.7 seconds. At this moment, the left leg touches ground first, the system starts to tilt to the right and the level controller responds by retracting the left leg and extending the right to compensate for the ground slope and return the base to the horizontal position. The LC trigger shows the moment the system detects landing and the signal that activates the level controllers is set on (set to 1). The graph shows the correlation between the simulation and the practical results. The prototype system reacts slower compared to the simulated system. The rate of descent, the system's inertia and also other inherent dynamics existing within the system could contribute to the outcome.

It can also be observed that in the simulations both legs retract/expand the same distance, but in the experimental test the height movement of the left leg is higher than the right leg.
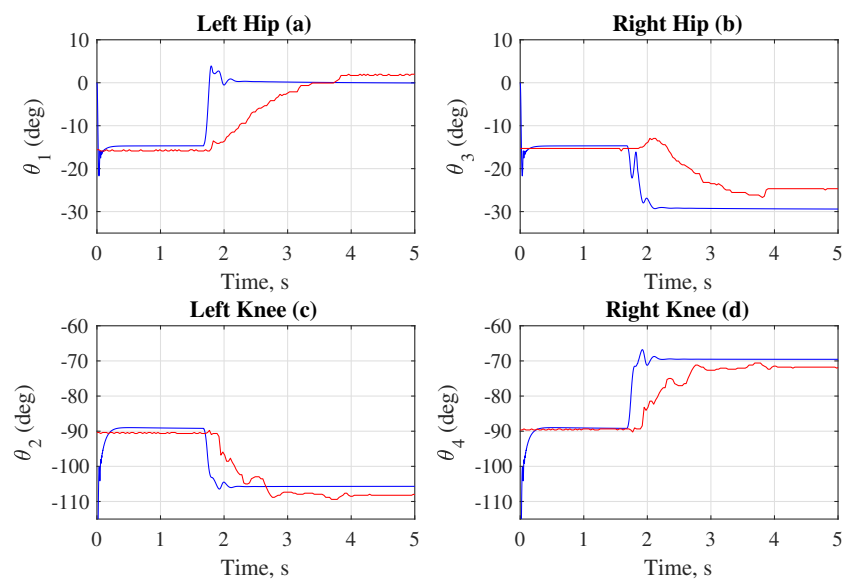

Figure 6. Joint angles at both hips and knees

Figure 6 shows the change of the legs' position in terms of joint angle variation. There is a clear evidence of correlation between the simulation and the practical results. It also shows that the practical system requires time to settle down before reaching stability. The retracting motion of the left leg is carried out by reducing $\theta_{1}$ and increasing $\theta_{2}$ and vice versa for the right leg. The results are shown to be consistent and repeatable.

It can be observed again how in the experimental test the left leg position changes more than in the simulations. In the right leg, the position variation is higher in the simulation than in the experimental test.
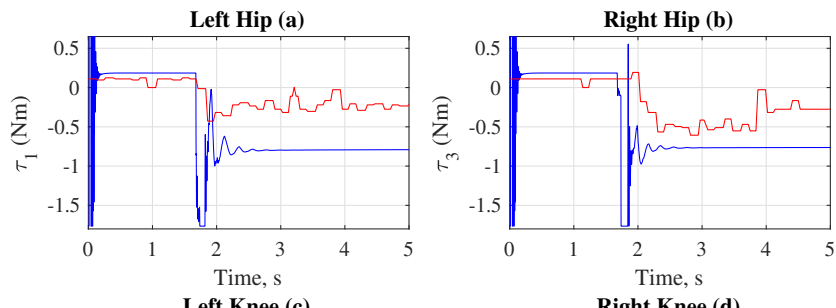

Left Knee (c)
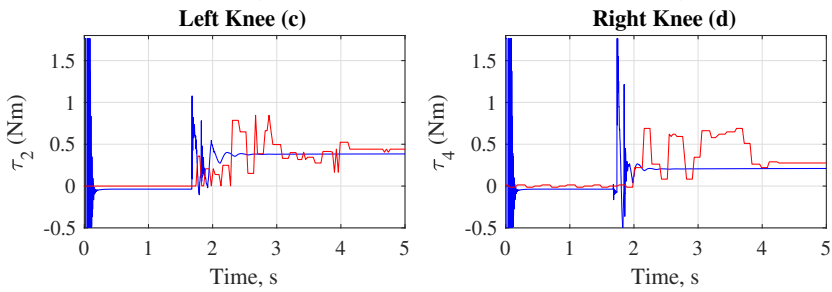

Figure 7. Joint torques at both hips and knees

Figure 7 shows the joint torques calculated in the software simulations compared with the load feedback from the servo motors. The manufacturer of the servos explicitly says that the load feedback is inaccurate and should be used only as an indicator of the direction and size of the torque applied [19], as it can be observed in the graphs. Despite that, the load feedback shows the weight of the system being transferred to the joints and how it changes direction at the moment of landing.

\section{Results Analisis}

First thing to notice is that the events in the simulations happen at much faster pace than in the experimental test. The level controller for example, corrects the inclination in less than 1 second, but in the prototype case it takes longer than 2 seconds, and the legs move much quicker in the simulations. One reason for that are the physical limitations of the hardware. Landing is a critical operation and therefore a controller that works at high frequency is desired in order to react faster to changes. This was the reason to choose a cycle time of $1 \mathrm{~ms}(1 \mathrm{kHz})$ in the simulations, but due to limitations of the microcontroller speed the cycle time in the experimental tests couldn't by reduced more than 30ms (33 $\mathrm{Hz}$ ). During the experimental tests the controller parameters have been increased to $K_{P}=0.018$ and $K_{I}=0.18$ to make the $L C$ react faster but the response time is still slower than the simulations.

Another reason is that the experimental test starts with the level controller off. This is because during the descent, the inclination of the base is not exactly 0 , but it changes around $\pm 1^{\circ}$, and the controller is switched off to avoid constant correction of the legs height before landing moment. The controller is turned on only when the landing moment is detected by the servos load feedback. This introduces a small 
delay in the experimental test, while in the simulations the level controller reacts instantaneously.

The rate of descent is another possible cause of the differences between the simulations and the real tests. In the simulations this parameter can be set, but in the experiments there was no measurement or control of the descent rate, and different speeds will produce different impact forces.

Despite the inaccuracy of the load feedback, it can be used to detect a change in the torque, and it has been successfully implemented to detect the moment of landing. By contrast, it cannot be used to calculate the real torque applied at the joint.

\section{CONCLUSIONS}

In this paper, a nonlinear dynamic model of a helicopter landing gear has been designed, simulated and compared to a laboratory-built prototype. The model includes the kinematic and dynamic equations, a landing scenario, including a controller to regulate the rate of descent and a ground contact model to simulate the ground-leg interaction, and a level controller to maintain the stability of the base when landing on irregular terrains. The results show a correlation between the simulations and the experimental tests, showing that the mathematical model is a good representation of the real system. The performance of the level controller in both cases is satisfactory as it maintains the stability of the helicopter while landing in slopped terrain.

The dynamic model presented in this paper constitutes a flexible tool that can be used to test different landing conditions as dimensions and masses of the bodies can be easily changed, as well as the landing speed and the type and condition of the terrain.

\section{REFERENCES}

[1] S. H. Mason, "Helicopter self-leveling landing gear". United States Patent US 3857533 A, 1974.

[2] D. W. Felder, "Slope Landing Compensator System". Patent US4062507 A, 1977.

[3] DARPA, "Robotic Landing Gear could enable future helicopters to take off and land almost everywhere," October 2015. [Online]. Available: http://www.darpa.mil/news-events/2015-09-10.

[4] V. Manivannan, J. P. Langley, M. F. Costello and M. Ruzzene, "Rotorcraft Slope Landings with Articulated Landing Gear," in AIAA Atmospheric Flight Mechanics (AFM) Conference, 2013.
[5] E. Leylek, M. Ward and M. Costello, "Flight Dynamic Simulation for Multibody Aircraft Configurations," Journal of Guidance, Control and Dynamics, vol. 35, pp. 1828-1842, 2012.

[6] X. Zhang, L. Lang, J. Wang and H. Ma, "The Quadruped Robot Locomotion Based on Force Control," in 27th Chinese Control and Decision Conference (CCDC), 2015.

[7] B. U. Rehamn, M. Focchi, J. Lee, H. Dallali, D. G. Caldwell and C. Semini, "Towards a Multi-legged Mobile Manipulator," in IEEE International Conference on Robotics and Automation (ICRA), Stockholm, Sweden, 2016.

[8] C. Korpela, M. Orsag and P. Oh, "Towards Valve Turning using a Dual-Arm Aerial Manipulator," in International Conference on Intelligent Robots and Systems (IROS), Chicago, IL, USA, 2014.

[9] J. Mendoza-Mendoza, G. Sepulveda-Cervantes, C. Aguilar-Ibanez, M. Mendez, M. Reyes-Larios, P. Matabuena and J. Gonzalez-Avila, "AirArm: A new kind of flying manipulator," in Workshop on Research, Education and Development of Unmanned Aerial Systems (REDUAS), Cancun, Mexico, 2015.

[10] B. Yuksel, G. Buondonno and A. Franchi, "Differential Flatness and Control of Protocentric Aerial Manipulators with Any Number of Arms and Mixed Rigid-/Elastic Joints," in International Conference on Intelligent Robots and Systems (IROS), Daejeon, Korea, 2016.

[11] M. W. Spong, S. Hutchinson and M. Vidyasagar, Robot Modelling and Control, John Wiley \& Sons, Inc, 2006.

[12] K. Goh, D. Melia Boix, J. McWhinnie and G. Smith, "Control of Rotorcraft Landing Gear on Different Ground Conditions," in IEEE International Conference on Mechatronics and Automation (ICMA 2016), Harbin, China, 2016.

[13] M. F. Velasquez-Lobo, J. M. Ramirez-Cortes and J. d. J. RangelMagdaleno, "Modeling a Biped Robot on Matlab/SimMechanics," in International Conference on Electronics, Communications and Computing, 2013.

[14] D. W. Marhefka and D. E. Orin, "Simulation of Contact Using a Nonlinear Damper Model," in International Conference on Robotics and Automation, Minneapolis, 1996.

[15] D. Naef, "Quadruped walking/running simulation," Swish Federal Institute of Technology, Zurich, 2011.

[16] B. Raiszadeh and D. Way, "Ground Contact Model for Mars Science Laboratory Mission Simulations," in AIAA Atmospheric Flight Mechanics Conference, Minneapolis, Minnesota, 2012.

[17] Y. Liu, J. Li, Z. Zhang, X. Hu and W. Zhang, "Experimental Comparison of five friction models on the same test-bed of the micro stick-slip motion system," Mechanical Sciences. Open Access, vol. 6, pp. 15-28, 2015.

[18] K. Ogata, Modern Control Engineering, 5th edition, New Jersey: Pearson Education, Inc, 2010.

[19] ROBOTIS, Dynamixel AX-12. User's Manual, 2006.

[20] S. M. Calvert, "Telescoping Landing Leg System". US Patent US9033276 B1, 19 May 2015. 